\title{
Toroidal dipole excitations in metamolecules formed by interacting plasmonic nanorods
}

\author{
Derek W. Watson, Stewart D. Jenkins, and Janne Ruostekoski \\ Mathematical Sciences and Centre for Photonic Metamaterials, University of Southampton, Southampton SO17 1BJ, United Kingdom
}

\author{
Vassili A. Fedotov and Nikolay I. Zheludev \\ Optoelectronics Research Centre and Centre for Photonic Metamaterials University of Southampton, \\ Southampton SO17 1BJ, United Kingdom
}

(Received 13 November 2015; revised manuscript received 3 February 2016; published 16 March 2016)

\begin{abstract}
We show how the elusive toroidal dipole moment appears as a radiative excitation eigenmode in a metamolecule resonator that is formed by pairs of plasmonic nanorods. We analyze one such nanorod configuration —a toroidal metamolecule. We find that the radiative interactions in the toroidal metamolecule can be qualitatively represented by a theoretical model based on an electric point dipole arrangement. Both a finite-size rod model and the point dipole approximation demonstrate how the toroidal dipole moment is subradiant and difficult to excite by incident light. By means of breaking the geometric symmetry of the metamolecule, the toroidal mode can be excited by linearly polarized light and appears as a Fano resonance dip in the forward scattered light. We provide simple optimization protocols for maximizing the toroidal dipole mode excitation. This opens up possibilities for simplified control and driving of metamaterial arrays consisting of toroidal dipole unit-cell resonators.
\end{abstract}

DOI: 10.1103/PhysRevB.93.125420

\section{INTRODUCTION}

The static toroidal dipole, also known as anapole, was first considered by Zel'dovich in 1957 [1]. The toroidal dipole has since been acknowledged in nuclear and atomic physics, where it is held responsible for parity violations in electroweak interactions [2-6], as well as in solid-state physics, where it leads to a special type of ordering in multiferroics [7-10].

The notion of the tororidal dipole was eventually extended to the dynamic case, where it generated a whole family of radiating toroidal multipoles [2,11-13], commonly omitted from standard literature on the electrodynamics [14-16]. While electric and magnetic multipoles are respectively linked to the oscillations of charges and transverse currents, the toroidal multipoles result from the oscillations of radial currents, which tend to be neglected in the long wavelength limit $[11,15]$. The lowest-order toroidal multipole-toroidal dipole-is produced by currents circulating on a surface of an imaginary torus along its meridians [17].

The toroidal response in natural materials is weak and usually masked by conventional electromagnetic (EM) effects, but with the advent of metamaterials and modern nanofabrication technology, materials composed of subwavelength particles may be designed and constructed to exhibit an enhanced toroidal dipole response. Various metamaterial designs have already been utilized experimentally to promote a toroidal dipole response in the microwave and optical part of the spectrum: circular apertures in a metallic screen [18], asymmetric split rings [19], split rings [20,21], and double bars [22]. In numerical simulations, also other resonator configurations [23-27] have shown notable toroidal dipole responses.

Thus far, the theoretical understanding of the toroidal dipole response in resonator systems has been limited and the conditions under which the toroidal moment may be excited on the microscopic level have not been well known. In this paper, we show theoretically how a simple structure formed by interacting plasmonic nanorods can support a collective excitation eigenmode that corresponds to a radiating toroidal moment. The toroidal mode is subradiant which could be important, e.g., for the applications of the toroidal moments in nonlinear optics $[28,29]$ and in surface plasmon sensors [30]. We analyze the light-induced interactions between the closely spaced plasmonic rods using a finite-size rod model as well as a model where the metamolecule is represented by a simple arrangement of point electric dipole emitters. We find that the point dipole model provides an accurate description of the radiative properties, except at very short inter-rod separations.

The generally weak coupling of the toroidal dipoles to external radiation fields makes it difficult to excite the toroidal mode. The structural symmetry of the mode inhibits the coupling to EM field modes that do not possess a similar "vortexlike" symmetry. We show how a simple linearly polarized incident light beam, however, can drive the toroidal dipole excitation when the geometric symmetry of the metamolecule is broken. The method is related to the double-resonator configuration introduced in Ref. [22] and we provide simple optimization protocols for maximizing the toroidal dipole mode excitation. The emergence of the toroidal dipole excitation is shown in the forward scattered light as a Fano resonance, indicating a destructive interference between the broad-resonance electric dipole and narrow-resonance toroidal dipole modes. Using linearly polarized beams for the excitation of the toroidal dipole mode can be especially beneficial in driving and controlling large metamaterial arrays of toroidal unit-cell resonators, since the coupling in this case is independent of the array or the beam symmetries.

The structure of this paper is as follows: in Sec. II, a brief review of metamaterial dynamics as formulated in Ref. [31] is presented. In Sec. III, the general model is applied to the case of cylindrical nanorods. Two models are developed: a point dipole model; and a finite-size resonator model. In Sec. IV, we investigate a toroidal metamolecule comprised of plasmonic nanorods. The collective radiative excitation eigenmodes, and their resonance linewidths and line shifts for the toroidal metamolecule are calculated. We then show how a toroidal dipole response may be excited with linearly-polarized light 
by introducing a geometric asymmetry in the metamolecule. Some concluding remarks are included in Sec. V.

\section{DYNAMICS OF METAMATERIAL SYSTEMS}

In order to analyze the EM interactions between plasmonic rods in a toroidal metamolecule and the excitation of toroidal dipole mode by an incident field we first briefly introduce the basic formalism of radiatively coupled rods. We show how in a simple model the incident field and the scattered EM fields from the other rods excite normal modes of current oscillations in each rod. A more detailed derivation of the formalism, also including magnetodielectric systems, can be found in Ref. [31].

In the general model of circuit resonator interactions with EM fields, we assume that the charge and current sources are driven by an incident electric displacement field $\mathbf{D}_{\text {in }}(\mathbf{r}, t)$, and magnetic induction $\mathbf{B}_{\text {in }}(\mathbf{r}, t)$ with frequency $\Omega_{0}$. When analyzing the EM fields and resonators, we adopt the rotating wave approximation where the dynamics is dominated by $\Omega_{0}$. In the rest of the paper, all the EM fields and resonator amplitudes refer to the slowly-varying versions of the positive frequency components of the corresponding variables, where the rapid oscillations $e^{-i \Omega_{0} t}$ due to the dominant laser frequency has been factored out.

The state of current oscillation in the resonator $j$ may be described by a single dynamic variable with units of charge $Q_{j}(t)$ and its rate of change $I_{j}(t)$, the current. The current oscillations within each resonator $j$ behave in a manner analogous to an LC circuit with resonance frequency $\omega_{j}$ :

$$
\omega_{j}=\frac{1}{\sqrt{L_{j} C_{j}}},
$$

where $C_{j}$ and $L_{j}$ are an effective self-capacitance and selfinductance, respectively. In Ref. [31], a general theory was formulated to derive a coupled set of linear equations for the EM fields and strongly coupled resonators. In order to express the coupled equations for the EM fields and resonators, we introduce the slowly varying normal mode oscillator amplitudes $b_{j}(t)$,

$$
b_{j}(t)=\frac{1}{\sqrt{2 \omega_{j}}}\left[\frac{Q_{j}(t)}{\sqrt{C_{j}}}+i \frac{\phi_{j}(t)}{\sqrt{L_{j}}}\right] .
$$

Here, the generalized coordinate for the current excitation in the resonator $j$ is the charge $Q_{j}(t)$ and $\phi_{j}(t)$ represents its conjugate momentum. In the rotating wave approximation, the conjugate momentum is linearly-proportional to the current [31]. The dynamic variable in Eq. (2) can be used to describe a general resonator with both polarization and magnetization sources. The oscillation of $Q_{j}(t)$ and $I_{j}(t)$ within each resonator are proportional to the corresponding polarization $\mathbf{P}_{j}(\mathbf{r}, t)$ and magnetization $\mathbf{M}_{j}(\mathbf{r}, t)$ sources [31]. The electric $\mathbf{E}_{\mathrm{sc}, j}(\mathbf{r}, t)$ and magnetic $\mathbf{H}_{\mathrm{sc}, j}(\mathbf{r}, t)$ fields scattered by emitter $j$, are a result of the oscillating polarization and magnetization sources.

We approximate the resonators as cylinders (nanorods) with the radius $a$ and length $H_{j}$, see Fig. 1 . In this work, we only consider a single radius for all nanorods, see Appendix A. In Sec. IV B and IV C, we vary the length of each nanorod

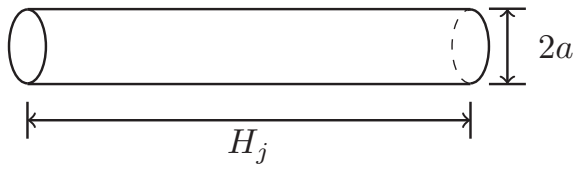

FIG. 1. Geometry of a single nanorod with length $H_{j}$ and radius $a$.

about a fixed length $H_{0}$. For simplicity, the charge and current oscillations within the cylinder are assumed to be linear along its axis. The magnetization of such a system is negligible $\mathbf{M}_{j}(\mathbf{r}, t) \simeq 0$. The scattered EM fields are thus determined by the polarization $\mathbf{P}_{j}(\mathbf{r}, t)$ within each nanorod alone, resulting in accumulation of charge on the ends of the rod.

The scattered electric field is then given by

$$
\mathbf{E}_{\mathrm{sc}, j}(\mathbf{r})=\frac{k^{3}}{4 \pi \epsilon_{0}} \int \mathrm{d}^{3} r^{\prime} \mathbf{G}\left(\mathbf{r}-\mathbf{r}^{\prime}\right) \cdot \mathbf{P}_{j}\left(\mathbf{r}^{\prime}\right),
$$

where $k=\Omega / c$. The radiation kernel $\mathbf{G}\left(\mathbf{r}-\mathbf{r}^{\prime}\right)$ determines the electric field at $\mathbf{r}$, from the polarization source at $\mathbf{r}^{\prime}$ [14]. The total electric fields external to resonator $j$ comprise the incident fields and those scattered from all other emitters,

$$
\mathbf{E}_{\mathrm{ext}, j}(\mathbf{r}, t)=\frac{1}{\epsilon_{0}} \mathbf{D}_{\mathrm{in}}(\mathbf{r}, t)+\sum_{j \neq j^{\prime}} \mathbf{E}_{\mathrm{sc}, j^{\prime}}(\mathbf{r}, t) .
$$

Equation (3) gives the total scattered electric field as a function of the polarization density, and is not readily solved for $\mathbf{P}(\mathbf{r}, t)$. When the relative separation between emitters is less than, or of the order of a wavelength, a strongly coupled system results.

We write the polarization density of the nanorod as [31]

$$
\mathbf{P}_{j}(\mathbf{r}, t)=Q_{j}(t) \mathbf{p}_{j}(\mathbf{r}),
$$

where we assume that the charge profile function $\mathbf{p}_{j}(\mathbf{r})$ may be considered to be independent of time. The driving of the charge oscillations within the nanorod is then provided by the external electric field $\mathbf{E}_{\text {ext }, j}(\mathbf{r}, t)$ aligned along the direction of the source, providing a net electromagnetic force (emf) [31], $\mathcal{E}_{\text {ext }, j}$,

$$
\mathcal{E}_{\mathrm{ext}, j}(t)=\frac{1}{\sqrt{\omega_{j} L_{j}}} \int \mathrm{d}^{3} r \mathbf{p}_{j}(\mathbf{r}) \cdot \mathbf{E}_{\mathrm{ext}, j}(\mathbf{r}, t) .
$$

For a system, which comprises $N$ resonators, the collective interactions of the resonators with each other and the external field is represented by the linear set of equations [31]

$$
\dot{\mathbf{b}}=\mathcal{C} \mathbf{b}+\mathbf{F}_{\text {in }},
$$

where $\mathbf{b}$ is a vector of normal oscillator variables and $\mathbf{F}_{\text {in }}$ is a vector describing the interaction of resonator $j$ with the incident EM field. The incident electric displacement field $\mathbf{D}_{\text {in }}(\mathbf{r}, t)$ with polarization vector $\hat{\mathbf{e}}_{\text {in }}$, is

$$
\mathbf{D}_{\text {in }}(\mathbf{r}, t)=D_{\text {in }} \hat{\mathbf{e}}_{\text {in }} e^{i k y} .
$$

The exact form of $\mathcal{C}$ depends on solving the scattered electric field, Eq. (3), for the polarization sources. The diagonal elements are a result of the resonator interacting with its selfgenerated EM fields, giving rise to the resonance frequency and decay rate of a single rod. The off-diagonal elements are the result of interactions between different resonators. 


\section{RESONATOR INTERACTIONS}

In the previous section, we briefly introduced the model for radiative coupling between different emitters that we will apply to the analysis of the toroidal metamolecule consisting of plasmonic rods. In this section, we focus on two approximations: a point emitter model and a finite-size model. The finite-size model is an extension of the previous treatment [31] and incorporates the corrections due to a finite-size polarization distribution that modifies the EM interactions between closely spaced rods. For the nanorods in Fig. 1, the electric field can be obtained using Eq. (3) with a suitable choice of the spatial profile of the charge excitations $\mathbf{p}_{j}(\mathbf{r})$.

\section{A. Point dipole approximation}

In the point dipole approximation, each resonator is modeled as a point electric dipole located at the center of mass of the resonator. The mode function $\mathbf{p}_{j}(\mathbf{r})$, of the electric dipole is defined as

$$
\mathbf{p}_{j}(\mathbf{r})=H_{j} \hat{\mathbf{d}}_{j} \delta\left(\mathbf{r}-\mathbf{r}_{j}\right),
$$

where the proportionality constant $H_{j}$ has units of length and the unit vector $\hat{\mathbf{d}}_{j}$ indicates the dipole orientation. The interaction of the resonator with its self-generated EM fields causes radiative damping to occur. The rate at which resonators radiate in the dipole approximation is $\Gamma_{\mathrm{E}, j}[31]$, where

$$
\Gamma_{\mathrm{E}, j}=\frac{C_{j} H_{j}^{2} \omega_{j}^{4}}{6 \pi \epsilon_{0} c^{3}} .
$$

We account for nonradiative losses by adding the phenomenological decay rate $\Gamma_{\mathrm{O}, j}$. The total decay rate is then the sum of the radiative emission rate and ohmic losses

$$
\Gamma_{j}=\Gamma_{\mathrm{E}, j}+\Gamma_{\mathrm{O}, j}
$$

The point dipole approximation, with the inclusion of magnetic dipoles, has previously been successfully applied to the studies of collective effects in planar resonator arrays, e.g., the transmission properties [32,33] and the development of an electron-beam-driven light source from the collective response [34].

\section{B. Finite-size resonator model}

Next, we consider the scattering of EM fields from a collection of finite-size nanorods, described earlier (see Fig. 1), by integrating over a constant density of atomic dipoles over the nanorod's volume.

The scattered electric field from nanorod $j$ due to polarization sources alone is, using Eqs. (3) and (5),

$$
\mathbf{E}_{\mathrm{sc}, j}(\mathbf{r}, t)=\frac{Q_{j} k^{3}}{4 \pi \epsilon_{0}} \int \mathrm{d}^{3} r^{\prime} \mathbf{G}\left(\mathbf{r}-\mathbf{r}^{\prime}\right) \cdot \mathbf{p}_{j}\left(\mathbf{r}^{\prime}\right) .
$$

In the limit $a, H \ll \lambda$, the analytic solution for the scattered EM fields from the nanorod in the far field zone yields the result of an oscillating point dipole, with the corresponding radiative linewidth of a point dipole of Eq. (10). In Appendix A, we will

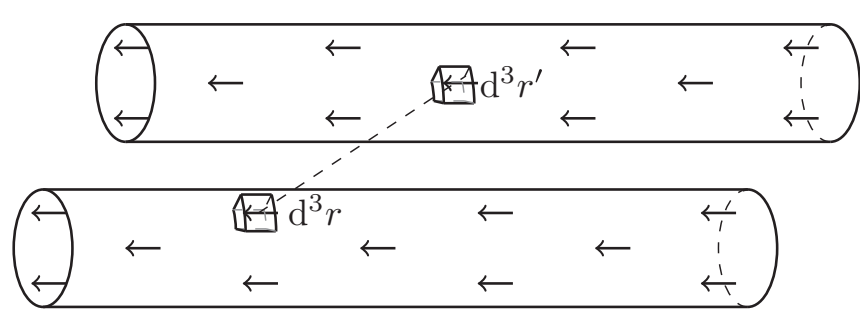

FIG. 2. Schematic of the interaction between two nanorods, which comprise a uniform distribution of electric dipoles (arrows) aligned along the axis of the cylinder.

describe how we estimate the radiative and ohmic decay rates for gold nanorods.

Whilst the far field radiation of a small nanorod behaves like that of a point dipole, the near and intermediate fields are much more complex, with the spatial dependence contributions varying as $1 / r^{3}$ and $1 / r^{2}$, respectively. The full field solution to Eq. (12) must be integrated numerically, also taking into account the finite thickness of the rods. The driving of nanorod $j$ is provided by an external emf Eq. (6), which can be broken into contributions from the incident and scattered EM fields

$$
\mathcal{E}_{\mathrm{ext}, j}=\mathcal{E}_{\mathrm{in}, j}+\sum_{j \neq j^{\prime}} \mathcal{E}_{j, j^{\prime}}^{\mathrm{sc}} .
$$

The incident emf $\mathcal{E}_{\text {in, } j}$ follows from Eq. (6) using the incident EM field Eq. (8). The emf driving the rod $j$ by the scattered fields from the rod $j^{\prime}, \mathcal{E}_{j, j^{\prime}}^{\mathrm{sc}}$, is obtained from Eqs. (6) and (12):

$$
\mathcal{E}_{j, j^{\prime}}^{\mathrm{sc}}=\frac{Q_{j^{\prime}} k^{3}}{4 \pi \epsilon_{0} \sqrt{\overline{\omega_{j} L_{j}}}} \int \mathrm{d}^{3} r \mathrm{~d}^{3} r^{\prime} \mathbf{p}_{j}(\mathbf{r}) \cdot \mathbf{G}\left(\mathbf{r}-\mathbf{r}^{\prime}\right) \cdot \mathbf{p}_{j^{\prime}}\left(\mathbf{r}^{\prime}\right) .
$$

Equation (14) consists of the integral over the volume of the nanorod located at $\mathbf{r}$ and the volume of the nanorod located at $\mathbf{r}^{\prime}$, see, e.g., Fig. 2.

\section{TOROIDAL METAMOLECULE}

In this section, we will use the theoretical models introduced in Secs. II and III to analyze the radiative interactions between plasmonic nanorods that form the toroidal metamolecule. We first show how a toroidal metamolecule composed of nanorods may exhibit a toroidal dipole response and calculate the eigenmodes of radiative excitations in the toroidal metamolecule. We then show how the toroidal metamolecule can be excited by incident EM fields when the symmetry of the metamolecule is broken.

Toroidal dipoles are formed by oscillating poloidal currents. The weakness of the toroidal dipole in comparison to its electric and magnetic counterparts in natural materials mean it is often neglected in classical physics [14-16].

We introduce a toroidal metamolecule that generally comprises $N$ nanorods distributed over two layers. Each layer has $N_{\theta}=N / 2$ emitters orientated radially outwards from the central axis of the metamolecule and equally spaced in the azimuthal direction. The second layer of emitters, identical to the first, is positioned a distance $2 y$ above the first layer. The orientation of both layers is such that there are $N_{\theta}$ pairs 
of parallel emitters. The symmetry of the metamolecule is therefore $C_{\frac{N}{2} h}$, a combination of $N / 2$ rotations about the $N / 2$-fold symmetry axis $C_{\frac{N}{2}}$ and the reflection in a horizontal plane $\sigma_{h}$ (a plane perpendicular to the principal axis of rotation).

In Cartesian coordinates, the orientation vectors of the nanorods are $\hat{\mathbf{d}}_{j}=\hat{\mathbf{x}} \cos \theta_{j}+\hat{\mathbf{z}} \sin \theta_{j}$. The density of the metamolecule is determined by $\Delta \theta=\theta_{j+1}-\theta_{j}$. As $\Delta \theta \rightarrow 0$, the metamolecule approaches a torus.

In the present section, for simplicity, we analyze the radiative properties of a toroidal metamolecule consisting of $N=8$ nanorods, where $\Delta \theta=\pi / 2$. Although an eigenmode of such a metamolecule only approximately describes a toroidal dipole, the characteristic properties of the resonator interactions and the toroidal dipole excitation by an incident field are already evident. As the density of the structure increases, the analysis can be easily extrapolated to account for the increased number of resonators as illustrated in the toroidal mode excitation shown in Figs. 8(a) and 8(b).

We first study a symmetric toroidal metamolecule (where all the rods are of equal length) and then break the geometric symmetry of the metamolecule in order to excite the toroidal mode using a simple light beam. In the symmetric metamolecule, we identify the associated collective modes of current oscillation and compare the resonance linewidths and line shifts obtained both in the point dipole approximation and in the finite-size resonator models. Finally, we determine how the toroidal dipole response of a toroidal metamolecule with some inherent asymmetry may be driven by linearly polarized light.

\section{A. Eigenmodes of a symmetric toroidal metamolecule}

A schematic illustration showing the arrangement and labeling system for the nanorods is shown in Fig. 3. In Cartesian coordinates, the locations of the point dipole and

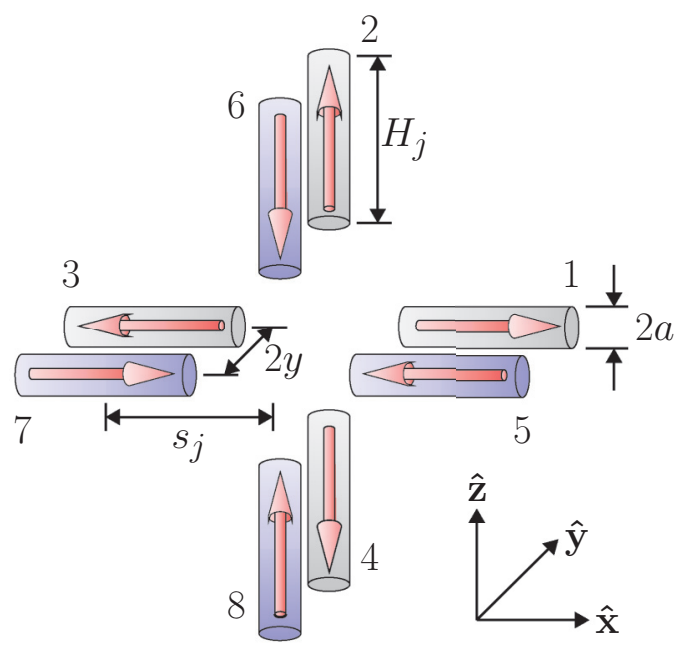

FIG. 3. Toroidal dipole mode of an eight symmetric rod metamolecule. The shading of the rods indicates those rods in a shared plane, the arrows indicate the phase of current oscillation. The radial position of the center of mass for an individual $\operatorname{rod}$ is $s_{j}$. The separation between parallel layers is $2 y$. the center of mass of the finite-size nanorods are

$$
\begin{aligned}
& \mathbf{r}_{1,5}=\left[\begin{array}{c}
s_{1,5} \\
\pm y \\
0
\end{array}\right], \quad \mathbf{r}_{2,6}=\left[\begin{array}{c}
0 \\
\pm y \\
s_{2,6}
\end{array}\right], \\
& \mathbf{r}_{3,7}=\left[\begin{array}{c}
-s_{3,7} \\
\pm y \\
0
\end{array}\right], \quad \mathbf{r}_{4,8}=\left[\begin{array}{c}
0 \\
\pm y \\
-s_{4,8}
\end{array}\right] .
\end{aligned}
$$

In a symmetric system then $s_{j}=s$ (for all $j$ ). Because the nanorods are symmetric, the radiative emission rate, ohmic loss rate and total decay rate of each rod are identical, i.e., $\Gamma_{\mathrm{E}, j}=\Gamma_{\mathrm{E}}, \Gamma_{\mathrm{O}, j}=\Gamma_{\mathrm{O}}$, and $\Gamma_{j}=\Gamma$. In Appendix A, we calculate the resonance frequency $\omega_{0}$ and relative decay rate $\Gamma_{\mathrm{O}} / \Gamma_{\mathrm{E}}$ as a function of the rod length for a finite-size rod using formulas for resonant light scattering from metal particles developed in Ref. [35], where ohmic losses are incorporated in the analysis by the Drude model. In order to simplify the comparisons between the point dipole approximation and the finite-size model, we use in the both models the same values for the decay rates. We choose the parameters for the gold nanorods of the symmetric metamolecule so that the length $H_{0}=1.5 \lambda_{p} \simeq 209 \mathrm{~nm}$, where $\lambda_{p}$ denotes the plasma wavelength for gold (see Appendix A). This yields $H_{0} \simeq$ $0.243 \lambda_{0}$ and $a \simeq 0.0324 \lambda_{0}$, where $\lambda_{0}=2 \pi c / \omega_{0} \simeq 859 \mathrm{~nm}$ denotes the resonance wavelength of the nanorod. We use these dimensions as those of a reference nanorod throughout the paper. The corresponding decay rates are $\Gamma_{\mathrm{E}} \simeq 0.83 \Gamma$ and $\Gamma_{\mathrm{O}} \simeq 0.17 \Gamma$, see Fig. 12 and Appendix A. The choice of these parameters ensures that the decay rates are only weakly sensitive to the small changes of the rod length.

The incident light, tuned to the resonance frequency of the nanorods $\Omega_{0}=\omega_{0}$, drives the charge oscillations within the nanorods. Each nanorod scatters light due to its polarization density that results from the charge oscillations. The light can multiply scatter between different resonators. Strong multiple scattering results in collective excitation modes in the system of nanorods. The collective eigenmodes can then exhibit different resonance frequencies and linewidths and line shifts [31-33]. We calculate the collective eigenmodes of the interacting rod configuration by analyzing the light-induced interactions in the equations of motion for the resonator excitations, Eq. (7). The collective modes of current oscillation within the system are described by the eigenvectors $\mathbf{v}_{n}$ of the interaction matrix $\mathcal{C}$. The corresponding eigenvalues $\xi_{n}$ have real and imaginary parts corresponding to the decay rate and resonance frequency shift of the mode,

$$
\xi_{n}=-\frac{\gamma_{n}}{2}-i\left(\Omega_{n}-\Omega_{0}\right) .
$$

The number of individual resonators determines the number of collective modes. The different modes may have superradiant or subradiant characteristics. The former occurs when the emitted radiation is enhanced by the interactions of the resonators $\left(\gamma_{n}>\Gamma\right)$, the latter occurs when the radiation is suppressed $\left(\gamma_{n}<\Gamma\right)$.

The classification of the different eigenmodes of the toroidal metamolecule comprising of symmetric nanorods is shown in Fig. 4. In Table I, we list the character table for the symmetry group $C_{n h}$ (for $n=4$ ) [36,37], where the eigenmodes are 
(a)

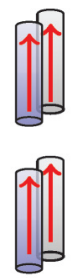

(c)
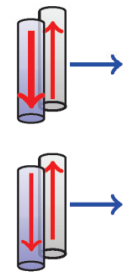

(e)

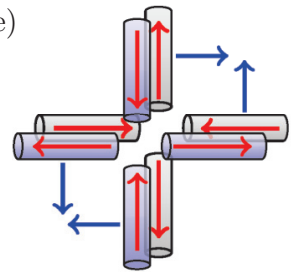

(g)

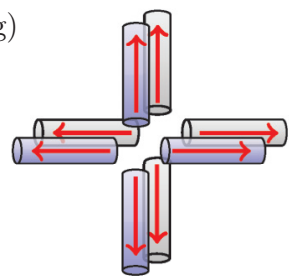

(b)

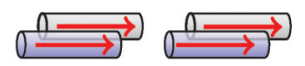

(d)

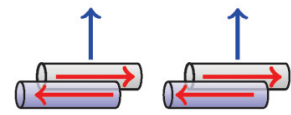

(f)

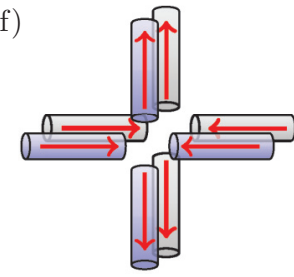

(h)

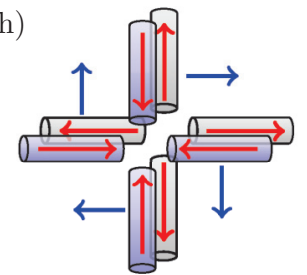

FIG. 4. Representation of the eigenmodes of a symmetric eightrod metamolecule. The red arrows represent the electric dipole moments and the blue arrows effective magnetic dipole moments. The modes are classified as (a) vertical and (b) horizontal electric dipole (E1) modes, (c) vertical and (d) horizontal magnetic dipole (B1) modes, (e) a magnetic quadrupole (B2) mode, (f) an electric quadrupole (E2) mode, (g) a symmetric (sy) mode, and (h) the toroidal dipole (t) mode. For shading and axis properties, see Fig. 3.

related to the corresponding Mulliken symbols of the point group.

The two modes depicted in Figs. 4(a) and 4(b) correspond to vertical and horizontal electric dipole (E1) modes, respectively. In the E1 modes, the responsive nanorods oscillate in phase and there is an effective electric dipole. Each of the E1 modes is a rotation of the other, consequently they experience the same resonance frequency shift and decay rate. In a similar manner to the E1 modes, Figs. 4(c) and 4(d) depict vertical and horizontal magnetic dipole (B1) modes, respectively. In the B1 modes, pairs of responsive nanorods oscillate out of phase, and the metamolecule forms an effective magnetic dipole. The B1 modes are also rotations of each other, thus experience the same resonance frequency shift and decay rate.

The remaining modes are all independent. Figure 4(e) corresponds to a magnetic quadrupole (B2) mode. Each parallel pair of emitters oscillates out-of-phase, and four independent effective magnetic dipoles form. Figure 4(f) depicts an electric quadrupole (E2) mode. In this mode, parallel pairs of emitters oscillate in phase with each other, but out of phase with

TABLE I. Character table of $C_{n h}$, for $n=4$. The terms in parentheses are our physical multipole designation, see Fig. 4, for the equivalent Mulliken symbols.

\begin{tabular}{lrrrrrrrr}
\hline \hline$C_{4 h}$ & $E^{\mathrm{a}}$ & $C_{4}$ & $C_{2}$ & $C_{4}^{3}$ & $i^{\mathrm{b}}$ & $S_{4}^{3 \mathrm{c}}$ & $\sigma_{h}$ & $S_{4}{ }^{\mathrm{c}}$ \\
\hline $\mathrm{A}(\mathrm{sy})$ & 1 & 1 & 1 & 1 & 1 & 1 & 1 & 1 \\
$\mathrm{~B}(\mathrm{E} 2)$ & 1 & -1 & 1 & -1 & 1 & -1 & 1 & -1 \\
$\mathrm{E}(\mathrm{E} 1)$ & 2 & 0 & -2 & 0 & 2 & 0 & -2 & 0 \\
$\mathrm{~A}^{\prime}(\mathrm{t})$ & 1 & 1 & 1 & 1 & -1 & -1 & -1 & -1 \\
$\mathrm{~B}^{\prime}(\mathrm{B} 2)$ & 1 & -1 & 1 & -1 & -1 & 1 & -1 & 1 \\
$\mathrm{E}^{\prime}(\mathrm{B} 1)$ & 2 & 0 & -2 & 0 & -2 & 0 & 2 & 0 \\
\hline \hline
\end{tabular}

${ }^{\text {a Identity. }}$

${ }^{\mathrm{b}}$ Inversion.

${ }^{\mathrm{c}}$ Improper rotation $S_{n}=C_{n} \sigma_{h}$.

their opposite parallel pair. Four independent effective dipoles combine to form an effective electric quadrupole. Figure $4(\mathrm{~g})$ is the symmetric mode, where all nanorods oscillate in phase. The toroidal dipole mode is shown in Figs. 4(h) and 3. In this mode, parallel pairs of emitters oscillate out of phase in such a way that the effective magnetic dipoles form a circular loop. The orientations of the nanorods lead to the orientation vectors for their corresponding electric dipole moments: $\hat{\mathbf{d}}_{1,7}=\hat{\mathbf{x}}$, $\hat{\mathbf{d}}_{2,8}=\hat{\mathbf{z}}, \hat{\mathbf{d}}_{3,5}=-\hat{\mathbf{x}}$, and $\hat{\mathbf{d}}_{4,6}=-\hat{\mathbf{z}}$.

We calculate the collective eigenmodes of the dynamic system described by Eq. (7), using the point dipole approximation and the finite-size resonator model discussed in Secs. III A and III B. In Figs. 5-7, the line shifts and the corresponding resonance linewidths are shown, for the collective modes, as functions of the metamolecule varying parameters $s$ and $y$ (in the former the layer spacing is fixed at $y=\lambda_{0} / 6$ and in the latter the radial spacing is fixed at $\left.s=\lambda_{0} / 4\right)$. These modes are depicted in Figs. 4(a)-4(g), respectively.

We find that the point dipole model qualitatively agrees with the finite-size model. The agreement becomes very good for larger rod separations. The toroidal dipole mode and the symmetric mode are subradiant for all parameter values we considered. This is also true for the magnetic quadrupole mode, B2, except for some specific values of the rod positions. The toroidal dipole mode is always the most subradiant mode, indicating a very weak coupling to external light fields. Magnetic dipole and electric quadrupole modes can generally exhibit both superradiant or subradiant characteristics depending on the precise details of the metamolecule's construction, while the electric dipole modes are almost always superradiant.

Specifically, for small radial separation $s=\lambda_{0} / 6$ (and the layer separation is fixed at $y=\lambda_{0} / 6$ ), the superradiant modes, $\mathrm{E} 1$ and $\mathrm{B} 1$ [Figs. 5(b) and 6(a)], have decay rates $\gamma_{\mathrm{E} 1} \approx 1.8 \Gamma$ and $\gamma_{\mathrm{B} 1} \approx 1.15 \Gamma$. The toroidal dipole mode has $\gamma_{\mathrm{t}} \approx 0.3 \Gamma$. When $s \approx \lambda_{0} / 4$, the E2 mode also becomes superradiant $\left(\gamma_{\mathrm{E} 2} \approx 1.25 \Gamma\right)$, while the decay rates of the E1 and B1 modes reduce to $\gamma_{\mathrm{E} 1} \approx 1.5 \Gamma$ and $\gamma_{\mathrm{B} 1} \approx \Gamma$, respectively. Even for the larger separation the toroidal dipole mode is still strongly subradiant $\left(\gamma_{\mathrm{t}} \approx 0.4 \Gamma\right.$ at $s \approx \lambda_{0} / 4$ and $\gamma_{\mathrm{t}} \approx 0.6 \Gamma$ at $\left.s \approx \lambda_{0} / 2\right)$. For large radial positions, $s \approx \lambda_{0} / 2$, only the E1 and E2 modes are superradiant $\left(\gamma_{\mathrm{E} 1} \approx 1.2 \Gamma\right.$ and $\gamma_{\mathrm{E} 2} \approx 1.65 \Gamma$, respectively) and the B2 mode becomes subradiant $\left(\gamma_{\mathrm{B} 1} \approx 0.75 \Gamma\right)$.

When we reduce the layer spacing to $y<\lambda_{0} / 16$, with the radial separation fixed at $s=\lambda_{0} / 4$, several of the modes 

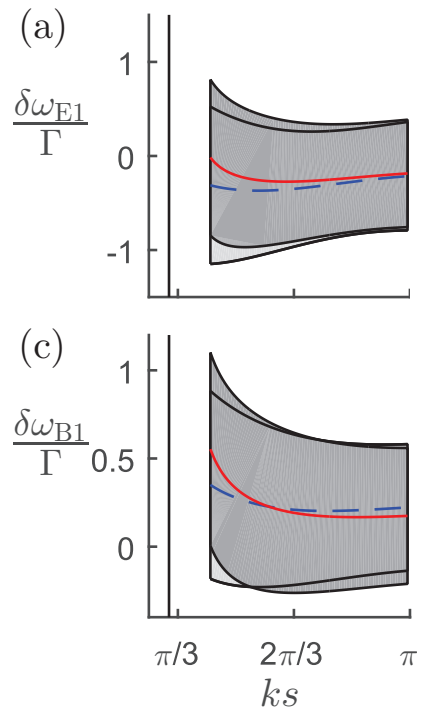

(b)

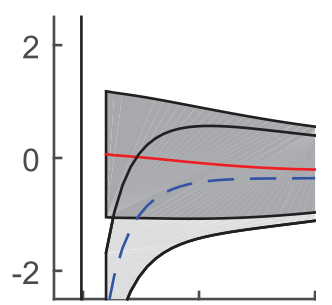

(d)

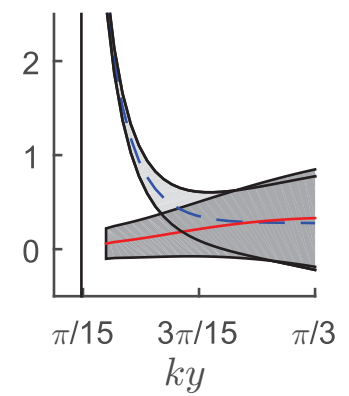

FIG. 5. The radiative resonance linewidths and line shifts for the collective electric dipole (E1) and magnetic dipole (B1) excitation eigenmodes, as a function of the metamolecule parameters $s$ and $y$. We show the line shift in the point dipole model (blue dashed line) and finite-size model (red solid line), the linewidth in the point dipole model (light shading about the blue dashed line), and the linewidth in the finite-size model (dark shading about the red solid line). In (a), the $\mathrm{E} 1$ mode, and in (c), the B1 mode, are shown as functions of the radial position $s$, with layer position $y=\lambda_{0} / 6$. In (b), the E1 mode, and in (d), the B1 mode, are shown as functions of the layer position $y$, with radial position $s=\lambda_{0} / 4$. The finite-size rods have lengths $H_{0}=0.243 \lambda_{0}$ and radii $a=0.0324 \lambda_{0}$. The radiative losses of each nanorod are $\Gamma_{\mathrm{E}}=0.83 \Gamma$, the ohmic losses are $\Gamma_{\mathrm{O}}=0.17 \Gamma$.

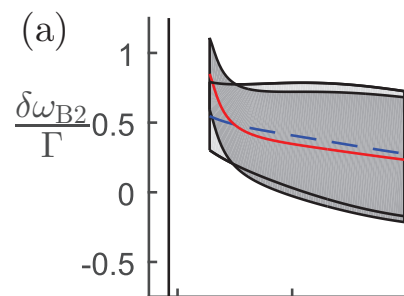

(b)
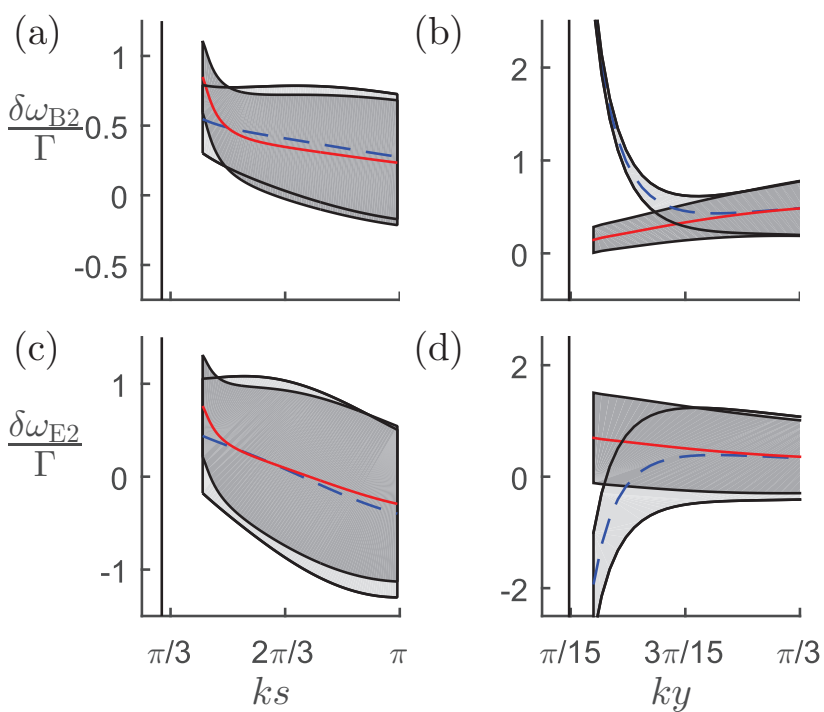

FIG. 6. The radiative resonance linewidths and line shifts for the collective magnetic quadrupole (B2) and electric quadrupole (E2) excitation eigenmodes, as a function of the metamolecule parameters $s$ and $y$. In (a), the B2 mode, and in (c), the E2 mode, are shown as functions of the radial position $s$. In (b), the B2, and in (d), the E2 mode, are shown as functions of the layer position $y$. For the rod parameters and plot descriptions see Fig. 5 caption.

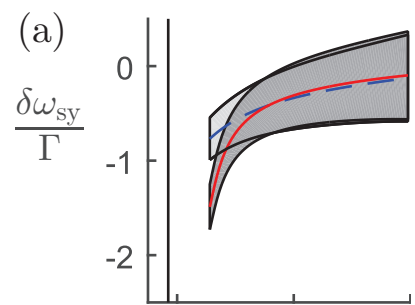

(b)
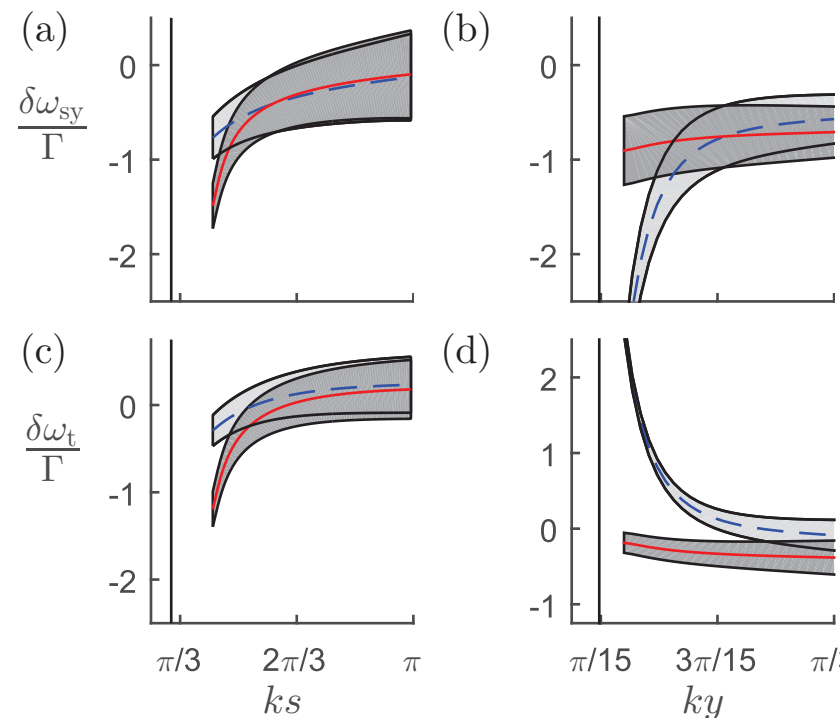

(d)

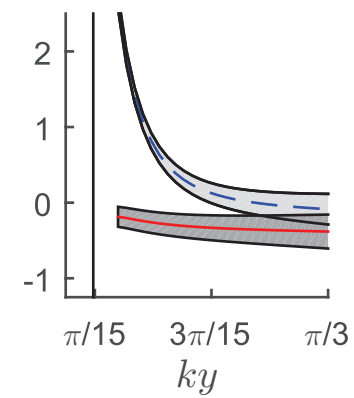

FIG. 7. The radiative resonance linewidths and line shifts for the collective symmetric and toroidal dipole excitation eigenmodes, as a function of the metamolecule parameters $s$ and $y$. In (a), the symmetric mode and (c) the toroidal dipole mode are shown as a function of the radial position $s$. In (b), the symmetric mode and (d) the toroidal dipole mode are shown as a function of the layer position $y$. For the rod parameters and plot descriptions see Fig. 5 caption.

become subradiant, except the $\mathrm{E} 1$ and $\mathrm{E} 2$ modes $\left(\gamma_{\mathrm{E} 1} \approx 1.6 \Gamma\right.$ and $\gamma_{\mathrm{E} 2} \approx 2.2 \Gamma$ at $y \approx \lambda_{0} / 16$ ). The toroidal dipole decay rate at $y \approx \lambda_{0} / 16$ is reduced to $\gamma_{\mathrm{t}} \approx 0.25 \Gamma$ from $\gamma_{\mathrm{t}} \approx 0.45 \Gamma$ at $y \approx \lambda_{0} / 6$.

Figures 5-7 also display the resonance line shifts of the modes, $\delta \omega_{n}=-\left(\Omega_{n}-\Omega_{0}\right)$. As the radial separation becomes large, these asymptotically approach a constant. This is due to the large separations resulting in the relatively close parallel pairs of nanorods interacting independently as dipoles. The B2 mode has the biggest shift with $\delta \omega_{\mathrm{B} 2} / \Gamma \approx 0.3$. The line shift of the toroidal dipole and $\mathrm{E} 1$ modes are small $\delta \omega_{\mathrm{t}} / \Gamma \approx 0.1$ and $\delta \omega_{\mathrm{E} 1} / \Gamma \approx 0.05$. As the separation becomes small, $s<\lambda_{0} / 4$, the line shifts of the finite-size model begins to deviate from those calculated in the point dipole approximation. At this range the total length of the metamolecule is $0.75 \lambda_{0}$ and finite lengths of the nanorods become increasingly important to their interactions.

When the layer spacing parameter $y$ is varied there is a more pronounced deviation in line shifts for small $y$. The line shift of the point dipole model begins to deviate when $y \approx 2 \lambda_{0} / 15$. Here the total width of the metamolecule is $\lambda_{0} / 6$, and the rods' finite radii begin to affect their interactions. In the region shown for $y$ in Figs. 5-7, the line shifts of the different modes do not approach constant values.

\section{B. Driving the toroidal dipole response}

A natural method of driving the toroidal dipole response in a toroidal metamolecule is to use radially polarized light. In the paraxial approximation, an incident displacement field $\mathbf{D}_{\text {in }}(\mathbf{r}, t)=D_{\text {in }}(\rho, y, t)(\cos \phi \hat{\mathbf{z}}+\sin \phi \hat{\mathbf{x}})$, can be estimated in terms of complex vectors $\hat{\mathbf{e}}_{+}$and $\hat{\mathbf{e}}_{-}$, where $\hat{\mathbf{e}}_{ \pm}=\mp(\hat{\mathbf{z}} \pm i \hat{\mathbf{x}}) / \sqrt{2}$, 
by

$$
\mathbf{D}_{\text {in }}(\mathbf{r}, t)=\frac{D_{\text {in }}(\rho, y, t)}{\sqrt{2}}\left[e^{i \phi} \hat{\mathbf{e}}_{-}-e^{-i \phi} \hat{\mathbf{e}}_{+}\right] .
$$

Equation (17) is a superposition of Laguerre-Gaussian beams with one unit of angular momentum which can couple directly to the toroidal dipole mode.

Employing radially polarized light, the toroidal dipole response may be driven in a symmetric toroidal metamolecule. When linearly polarized light is shone on the symmetric toroidal metamolecule the toroidal dipole response is suppressed; the dominant responses driven are the E1 responses.

Here, we analyze in detail how a toroidal dipole mode can also be excited using linearly polarized light and provide a simple protocol how to optimize the toroidal dipole excitation. Linear polarization has an advantage that it is readily available in an experiment and can easily be employed to drive toroidal dipole modes in large arrays of metamolecules, independently of the symmetry of the array or the beam.

Rather than spatially varying the light field to alter the excitation of individual rods (as done in the case of radial polarization) we alter the responses of individual rods to linearly polarized light by tailoring the length of the rods. Introducing the asymmetry in the rod lengths, according to Fig. 12(b), shifts the resonance frequencies and introduces a geometric asymmetry in the metamolecule. A similar principle was phenomenologically introduced in Ref. [22] where asymmetric pairs of nanorods were experimentally employed to produce a toroidal dipole response.

To see how the rods should be altered, we consider an incident linearly polarized light wave tuned to the resonance frequency $\Omega_{0}=\omega_{0}$ of our reference nanorod. The length of each rod $j$ is then changed by $\delta H_{j}$, while the radius is fixed. For sufficiently small $\delta H_{j}$, the alteration shifts the rod's resonance frequency by $\delta \omega_{j}$ in proportion to $\delta H_{j}$, as we demonstrate using the Drude model in Appendix A, see Fig. 12. In this section, we will derive the pattern of rod length asymmetries required for linearly-polarized light to excite a toroidal dipole in the limit that the incident field is far detuned from resonance with any individual nanorod, i.e., $\delta \omega_{j} \gg \Gamma_{j}$. In this limit, interactions between nanorods can be neglected. We demonstrate how this scheme functions with smaller asymmetries in the presence of interactions in Appendix B.

We assume that the two layers are separated by a distance much less than a wavelength, hence the phase difference of the incident field between layers is negligible. In order to couple the field to all nanorods, we choose the polarization of the incident field to be such that the it bisects the angle created by two adjacent nanorods in the same plane, as depicted in Fig. 8. For a symmetric metamolecule, a field propagating into the plane of the metamolecule induces an emf [described by Eq. (6)] driving each nanorod $j$ with an amplitude

$$
F_{\text {sym }, j}=F_{0} \cos \theta_{j} e^{i k y_{j}},
$$

where $F_{0}$ is the driving amplitude of a rod oriented parallel to the incident field polarization, and $y_{j}$ is the position coordinate of rod $j$ along the incident field's propagation direction. The strength of interaction between the driving field and a nanorod varies with the angle $\theta_{j}$ between the nanorod and polarization
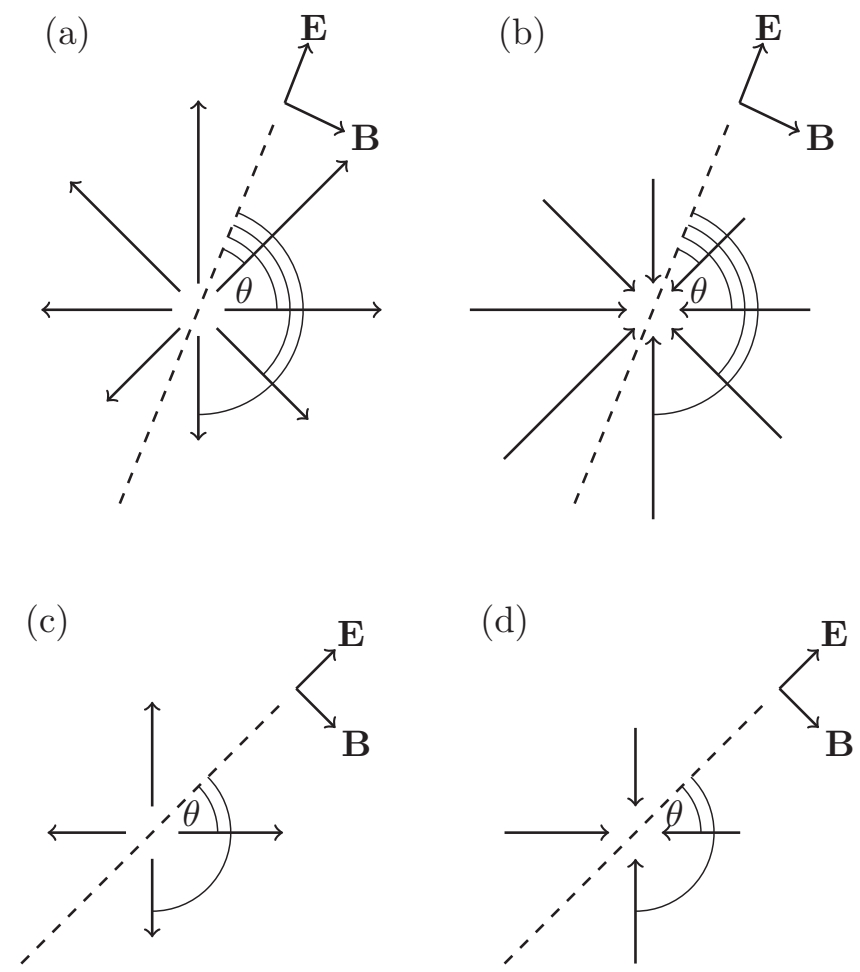

FIG. 8. The excitation of the toroidal dipole mode by linearly polarized light. The length of the arrows indicate the rod lengths which, together with the angle $\theta$ each rod makes with the polarization of the incident light, ensures each rod is equally excited. The arrow direction indicates the state of the current oscillation within each nanorod. In (a), the top layer and (b) the bottom layer of the more general $N=16$ case is shown. In (c), the top layer and (d) the bottom layer of the $N=8$ case is shown. The $N=8$ case is considered in the numerical simulation.

of the incident light. Because the emf induced by the incident field along a rod is proportional to its length, the asymmetry in rod lengths perturbs the driving strength of each $\operatorname{rod} j$ in proportion to $\delta H_{j}$, so that the rod driving is

$$
F_{j}=\left(F_{0}+\delta F_{j}\right) \cos \theta_{j},
$$

where $\delta F_{j} \propto \delta H_{j}$ is the change in driving amplitude rod $j$ would experience if it were parallel to the incident field.

Under these circumstances, when $\delta \omega_{j} \gg \Gamma_{j}$, interactions between resonators can also be ignored (i.e., $\delta \omega_{j} \gg \mathcal{C}_{j j^{\prime}}$ for $\left.j \neq j^{\prime}\right)$ in the dynamics of nanorod $j$, and

$$
\dot{b}_{j} \approx i \delta \omega_{j} b_{j}+\left(F_{0}+\delta F_{j}\right) \cos \theta_{j} .
$$

Thus, to lowest order in $\delta H_{j}$, nanorod $j$ has the steady-state response to the incident field

$$
b_{j} \simeq i \frac{\cos \theta_{j}}{\delta \omega_{j}} F_{0} .
$$

Therefore, in the noninteracting limit, one could engineer the response of a metamolecule simply by adjusting the resonance frequencies of its individual rods.

In a toroidal dipole excitation, all resonators in the layer $+y$ oscillate radially outward (inward) in phase with each 
other, while those resonators in the layer $-y$ oscillate radially inward (outward) in phase. Such an excitation corresponds to the toroidal dipole eigenmode of the completely symmetric metamolecule. This is indicated by the arrows in Fig. 3. To obtain this excitation profile, Eq. (21) suggests that the rod lengths should be modified such that

$$
\delta H_{j}=\left\{\begin{array}{cl}
\delta H_{0} \cos \theta_{j} & \text { for } j=1, \ldots, \frac{N}{2} \\
-\delta H_{0} \cos \theta_{j} & \text { for } j=\frac{N}{2}+1, \ldots, N
\end{array}\right.
$$

where $\delta H_{0}$ is a reference change in rod length, and we have assumed that $\delta H_{0}$ is sufficiently small that $\delta \omega_{j} \propto \delta H_{j}$. Although this profile of rod lengths was arrived at in a regime where interactions are neglected, we show that a similar distribution of lengths can also be effective in producing a toroidal dipole from a linearly-polarized light wave driving in Appendix B. We illustrate the dependence of a nanorod's length, and hence resonance frequency, on its position within the metamolecule for the cases of $N=16$ and $N=8$ nanorods in Fig. 8.

For a toroidal metamolecule, which comprises eight nanorods, only two distinct lengths (resonance frequencies) are required in order to produce a toroidal dipole excitation from linearly polarized light. This equates to a difference in rod length $\mp \delta H$ about a mean rod length $H_{0}$. We define $H_{l, s}=H_{0} \pm \delta H$ with $H_{1,2,7,8}=H_{l}$ and $H_{3,4,5,6}=H_{s}$, such that each parallel pair of rods is composed of a long and short rod, see Figs. 8(c) and 8(d), whose center's of mass are located at $s_{j}=s$. The polarization vector of the incident field, Eq. (8), that will excite the toroidal dipole mode is $\hat{\mathbf{e}}_{\mathrm{in}}=(\hat{\mathbf{x}}+\hat{\mathbf{z}}) / \sqrt{2}$. The eigenmodes and line shifts and widths for a symmetric toroidal metamolecule comprising rods with length $H_{0}$ were discussed in Sec. IV A. When the radial position of the reference rod is fixed, i.e., $s_{l}=\lambda_{0} / 3$, and some asymmetry in rod length is introduced, the response of the metamolecule becomes a function of the relative rod lengths $H_{s} / H_{l}$.

\section{Excitations of the toroidal dipole mode}

The eigenmodes in Fig. 4 are those of a symmetric toroidal metamolecule. When analyzing the amplitudes of the eigenmodes, of an asymmetric metamolecule, we do so using the symmetric metamolecule basis. The coupling matrix $\mathcal{C}$ is decomposed as

$$
\mathcal{C}=\mathcal{C}_{\text {sym }}+\mathcal{A}
$$

where $\mathcal{C}_{\text {sym }}$ is the coupling between rods whose lengths are the mean rod length $H_{0}$. The matrix $\mathcal{A}$ contains the detail on asymmetry. The variation of the resonance frequencies between the rods generally suppresses the light-mediated interactions in the metamolecule [38]. For the point dipole model, we define this as a diagonal matrix whose elements are the resonance frequency shifts of the different nanorods, see Appendix B. In the finite-size resonator model, in addition to the resonance frequency shifts in the diagonals, the off-diagonal elements give the difference in the finite-size resonator interactions of a symmetric system and an asymmetric system, see Appendix B.
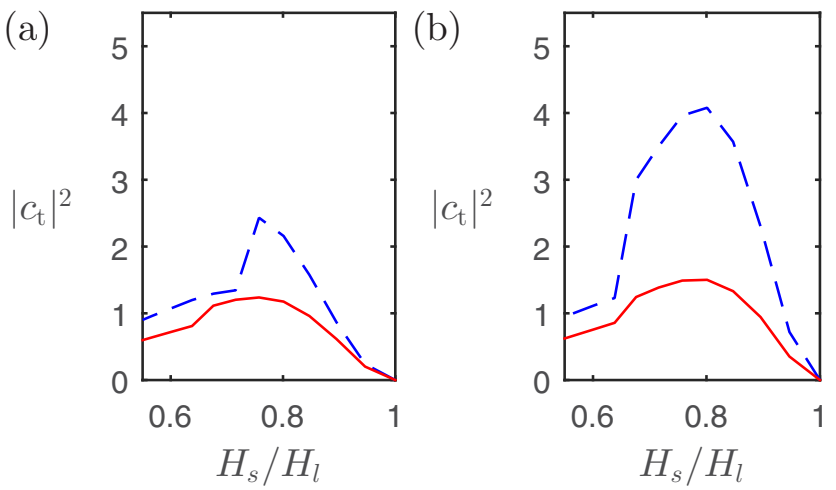

FIG. 9. The intensity of the collective toroidal dipole excitation as a function of the ratio of rod lengths $H_{s} / H_{l}$. The center of mass of the longer rods is at $s_{l}=\lambda_{0} / 3$ and the layer position is $y=\lambda_{0} / 10$. We show the point dipole model (blue dashed lines) and finite-size model (red solid lines). The nanorod mean length and radius are those of the reference nanorod. The radiative emission rate in (a) is $\Gamma_{\mathrm{E}}=0.83 \Gamma$. In (b), there are no ohmic losses.

The amplitude of the different modes may be analyzed by expanding the vector of dynamic variables $\mathbf{b}(t)$ as

$$
\mathbf{b}(t)=\sum_{n} c_{n}(t) \mathbf{v}_{n},
$$

where $c_{n}(t)$ is the amplitude of the eigenmode $\mathbf{v}_{n}$ of $\mathcal{C}_{\text {sym }}$. We denote the toroidal dipole amplitude as $c_{\mathrm{t}}$. In the absence of any asymmetry, the only modes driven by linearly polarized light are the E1 modes. When the asymmetry depicted in Fig. 8 is introduced to the metamolecule, the toroidal dipole mode (in addition to the E1 modes) is also driven.

In Fig. 9, we show the maximum intensity of the toroidal dipole mode as a function of the asymmetry between the cylindrical rods, for the point dipole approximation and for the finite-size model, when the metamolecule is driven at the resonance of the toroidal mode of the symmetric metamolecule. If there are no ohmic losses, the finite-size model shows a maximum intensity when $H_{s} / H_{l} \approx 0.8$, and the intensity here of the point dipole model is approximately four times that of the finite-size model. As the asymmetry between the rods increases, the intensity of both the finite-size model and the point dipole approximation decreases.

When ohmic losses are accounted for, the maximum intensity is when $H_{s} / H_{l} \approx 0.75$ before decreasing. The incorporation of losses significantly affects the point dipole approximation, the maximum intensity is approximately $50 \%$ less than when no losses were present. Conversely, the effect on the finite-size model is negligible.

In Fig. 10, the relative occupation of the toroidal dipole mode is shown as a function of the ratio of rod lengths. Although we consider a nonunitary, open system, the eigenmodes have periodic boundary conditions and in the studied cases form a well-behaving orthonormal pseudo-basis. We define the overlap between an eigenmode $\mathbf{v}_{j}$ with an excitation $\mathbf{b}$ by

$$
O_{j}(\mathbf{b}) \equiv \frac{\left|\mathbf{v}_{j}^{T} \mathbf{b}\right|^{2}}{\sum_{i}\left|\mathbf{v}_{i}^{T} \mathbf{b}\right|^{2}},
$$



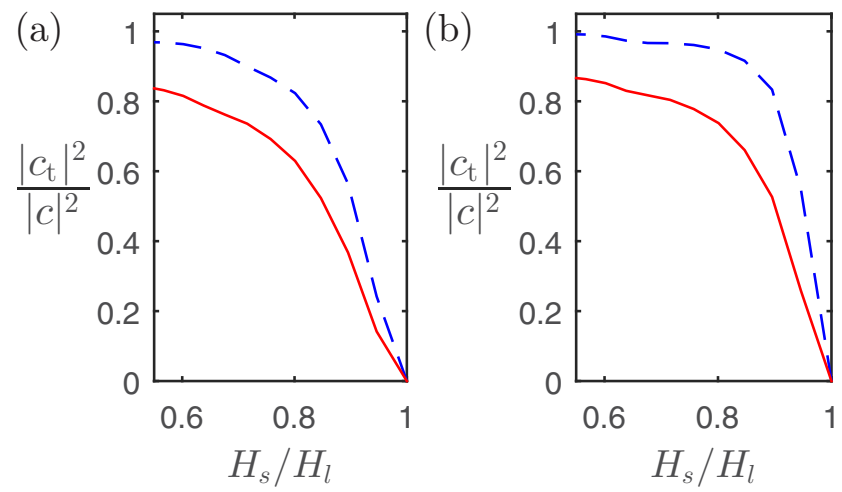

FIG. 10. The relative amplitude of the collective toroidal dipole excitation as a function of the ratio of rod lengths when driven on the toroidal dipole resonance. The parameters as in Fig. 9.

where the summation runs over all the eigenmodes. In both cases, when losses are present and when they are neglected, the relative occupation of the point dipole approximation overestimates the finite-size model. In the absence of ohmic losses, the relative occupation of the point dipole model saturates at $H_{s} / H_{l} \approx 0.8$, where the total excitation of the metamolecule is in the toroidal dipole mode. In the finite-size model when $\Gamma_{\mathrm{O}}=0$, saturation occurs when $H_{s} / H_{l} \approx 0.7$ and the relative occupation is approximately 0.8 . When losses are present, the relative occupation at the maximum intensity of the toroidal dipole excitation $\left(H_{s} / H_{l} \approx 0.75\right)$ is 0.95 for the point dipole approximation. The finite-size model here shows a relative occupation of 0.75 .

In the absence of asymmetry between the rods $H_{s} / H_{l}=1$, both the intensity plots in Fig. 9, and the relative occupation plots Fig. 10, show that there is no toroidal dipole excitation. However, even a small asymmetry in rod lengths produces a toroidal dipole excitation. Although the intensity of the toroidal mode excitation can be maximized at a relatively small value of the rod asymmetry, the fidelity of the toroidal dipole mode keeps increasing when the asymmetry is increased.

\section{Scattered light intensity in the far field}

It is also interesting to study the scattered light from a toroidal metamolecule in the far-field response. We again assume that the incident field propagating normal to the plane of the metamolecule excites the current oscillations in the nanorods. We calculate the collective excitations of the metamolecule by including all the radiative interactions between the nanorods. In Fig. 11, we show the intensity of the scattered light in the forward direction (without the incident field contribution) from a symmetric toroidal metamolecule and from a toroidal metamolecule with the asymmetry designed to promote the toroidal dipole response. Two cases are displayed corresponding to two layer separations $y$. In the symmetric case, only the E1 collective modes are excited, displaying broad resonances.

For large $y$, the E1 resonance is close to the resonance frequency, $\omega_{0}$ of our reference nanorod, for small $y$ the resonance is blue-shifted. In the asymmetric case, the light excites the E1 modes and the toroidal dipole mode. A destructive interference
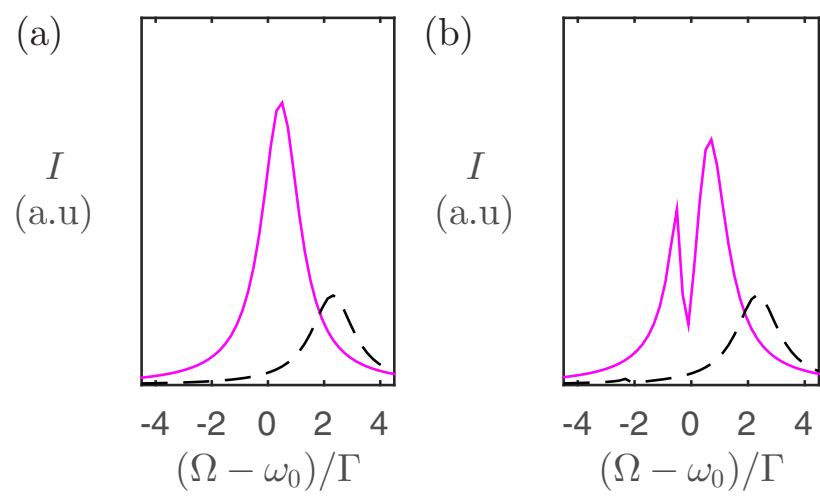

FIG. 11. The scattered light intensity in the forward direction $I$, as a function of the detuning of the incident light from the resonance frequency $\omega_{0}$ of our reference nanorod. We show the responses of a symmetric toroidal metamolecule (a) and a toroidal metamolecule with asymmetry promoting a toroidal response (b). The radial position of the nanorods $s=\lambda_{0} / 3$, the layer position $y=\lambda_{0} / 20$ (black dashed lines) and $y=\lambda_{0} / 10$ (magenta solid line), $H_{s} / H_{l}=0.75$, and $\Gamma_{\mathrm{E}}=0.83 \Gamma$. The intensity dip in (b) indicates a Fano resonance due to the interference between the E1 and toroidal dipole modes. The calculations are performed in the point dipole approximation.

between the broad-resonance E1 and the narrow-resonance toroidal modes produces a Fano resonance [39]. For the case of a large layer separation $y$, the Fano resonance clearly shows up as a dip in the spectrum of the scattered light intensity at the resonance of the toroidal dipole mode, indicating suppressed forward scattering. This is because a toroidal dipole excitation on a plane normal to the propagation direction does not contribute to the far-field radiation.

The interference of the Fano resonance has an analogy in atomic physics in the interference of bright and dark modes in the electromagnetically-induced transparency [40]. Here the subradiant toroidal mode $(\mathrm{t})$ acts as a dark radiative mode and the superradiant electric dipole mode (E1) as a bright mode; in the excitation of the E1 mode, the different scattering paths, $\rightarrow \mathrm{E} 1, \rightarrow \mathrm{E} 1 \rightarrow \mathrm{t} \rightarrow \mathrm{E} 1$, etc., destructively interfere at the Fano resonance peak. The Fano resonances may also appear in other complex metamolecules, such as in oligomers [41], or as a result of a collective behavior of the metamaterial array [33]. The existence of more than one subradiant mode in a toroidal metamolecule and the possibility to employ collective effects in ensembles of toroidal metamolecules is particularly promising for tunable control of the resonances [33] and for sensing applications.

Our simple model of the radiative intensity provides a qualitative description of the Fano resonance of the toroidal dipole mode in the forward-scattered far-field spectrum. For 2D metamaterial arrays of asymmetric split-ring metamolecules, the point dipole radiation model provides a good qualitative agreement with the experimental findings due to weak higher-order multipole radiation of individual split-ring arcs [32]. In the studied cases of the resonances of individual nanorods, higher-order multipole radiation is similarly weak. Comparisons between multipole expansions and complete 
field calculations were performed in Ref. [25] between the far-field radiation patterns of toroidal dipole resonances in a noninteracting resonator system.

\section{CONCLUSIONS}

We theoretically studied light-induced interactions in a toroidal metamolecule that consists of closely-spaced, strongly-coupled plasmonic nanorods. The interactions lead to collective excitation eigenmodes that exhibit collective resonance frequencies, linewidths, and line shifts. When the nanorod pairs are pointing radially outwards, one of the collective eigenmodes is identified as a toroidal dipole mode. We provided simple criteria to optimize a structural asymmetry of the metamolecule that allows a strong excitation of the toroidal dipole mode by a simple linearly-polarized light beam. By analyzing a specific eight-rod case, we have shown how even small asymmetries lead to a large proportion of the total excitation to be found in the toroidal dipole mode.

By comparing the point dipole approximation to a finitesize resonator model, we have shown that the point dipole approximation is sufficient to model interacting rods for large interrod separations, providing accurate descriptions when the layer and radial separations satisfy $y \gtrsim \lambda_{0} / 6$ and $s \gtrsim \lambda_{0} / 4$. For more closely-spaced rods, the nanorods' finite length and thickness become increasingly important.

\section{ACKNOWLEDGMENTS}

We acknowledge financial support from the EPSRC, the Leverhulme Trust, the Royal Society, and the MOE Singapore Grant No. MOE2011-T3-1-005.

\section{APPENDIX A: DRUDE MODEL}

In this Appendix, we consider the scattering and polarizability of small metallic nanorods in order to estimate the resonance frequency, as well as the radiative and ohmic decay rates of a single nanorod. We begin by considering the Drude model for the permittivity $\epsilon$ of a metallic rod [42],

$$
\epsilon(\omega)=\epsilon_{\infty}-\frac{\omega_{p}^{2}}{\omega\left(\omega+i \Gamma_{\mathrm{D}}\right)},
$$

where $\epsilon_{\infty}$ is the permittivity at infinite frequencies, $\omega_{p}$ is the plasma frequency and $\Gamma_{\mathrm{D}}$ is the decay rate of current oscillations within the material. The scattering cross section of a small particle is dependent upon its polarizability $\alpha$ [42] and the wavelength $\lambda$ of the incident field

$$
\sigma_{\mathrm{sc}}=\frac{8 \pi^{3}}{3 \lambda^{4}}|\alpha|^{2}
$$

The polarizability depends on the physical characteristics of the particle including its volume $V_{0}$ and geometry, which is introduced through the depolarization factor $L$ [42]. In the Rayleigh approximation, the polarizability is

$$
\alpha_{i}=V_{0} \frac{\epsilon-1}{1+L_{i}(\epsilon-1)}, \quad i=x, y, z
$$

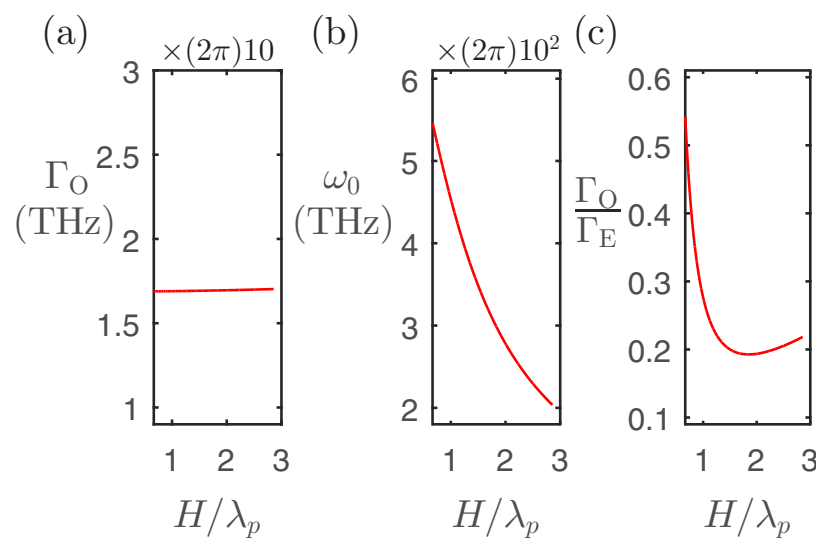

FIG. 12. The ohmic losses, resonance frequency, and relative radiative decay rate as a function of the rod length for a gold nanorod with radius $a=\lambda_{p} / 5$. We show the ohmic losses $\Gamma_{\mathrm{O}}$ in (a), the resonance frequency $\omega_{0}$ in (b) and the relative radiative decay rate $\Gamma_{\mathrm{O}} / \Gamma_{\mathrm{E}}$ in (c).

The depolarization factor for a cylinder aligned along the $z$ axis is $[43,44]$

$$
L_{x}=L_{y}=\frac{1}{2 \sqrt{1+\kappa^{2}}} \quad \text { and } \quad L_{z}=1-\frac{1}{\sqrt{1+\kappa^{2}}},
$$

where $\kappa=2 a / H$ is the aspect ratio of the cylinder. The curve produced by the scattering cross section Eq. (A2) has two Lorentzian profiles with two independent resonance frequencies. There is a resonance representing the longitudinal polarizability $\alpha_{z}$ with depolarization factor $L_{z}$, and a separate resonance for the radial polarizability $\alpha_{x}=\alpha_{y}$ with depolarization factors $L_{x}=L_{y}$.

For gold, the Drude parameters are [45-47] $\epsilon_{\infty}=9.5$, $\omega_{p}=(2 \pi) 2200 \mathrm{THz}$, and $\Gamma_{\mathrm{D}}=(2 \pi) 17 \mathrm{THz}$. In the Rayleigh approximation, the full width at half maximum (FWHM) of the scattering cross section Eq. (A2) is approximately independent of the length of the rod and gives the value of the ohmic loss rate $\Gamma_{\mathrm{O}}$. In Figs. 12(a) and 12(b), we show $\Gamma_{\mathrm{O}}$ and resonance frequency $\omega_{0}$ for a gold nanorod with radius $a=\lambda_{p} / 5$, where $\lambda_{p}=2 \pi c / \omega_{p} \simeq 139 \mathrm{~nm}$. We find $\Gamma_{\mathrm{O}} \simeq \Gamma_{\mathrm{D}} \simeq(2 \pi) 17 \mathrm{THz}$.

For larger particles, the Rayleigh approximation is insufficient and retardation effects must be considered. Mie's formulation accounts for this retardation for spherical particles. In Ref. [35], a generalization of Mie's polarizability is obtained for nonspheroidal particles that has been used successfully to model the scattering of metallic nanoparticles. The approximate ratio of ohmic losses to the radiative decay rate is

$$
\begin{aligned}
\frac{\Gamma_{\mathrm{O}}}{\Gamma_{\mathrm{E}}} & =-\operatorname{Im}\left(\frac{3 \lambda_{0}^{3}}{4 \pi^{3} a^{2} H(\epsilon-1)}\right) \\
& =\frac{6 \Gamma_{\mathrm{D}} \omega_{p}^{2} c^{3}}{a^{2} H \omega_{0}^{2}\left[\omega_{0}^{2} \Gamma_{\mathrm{D}}^{2}\left(\epsilon_{\infty}-1\right)+\left(\omega_{0}^{2}\left(\epsilon_{\infty}-1\right)-\omega_{p}^{2}\right)^{2}\right]}
\end{aligned}
$$

In Fig. 12(c), we show the relative decay rates as a function of the rod length. For shorter rods $H<\lambda_{p}$, the ohmic losses are dominant. For longer rods, the radiative emission rate is dominant. For $\lambda_{p}<H<3 \lambda_{p}$, the radiative decay rate is 
approximately constant, $\Gamma_{\mathrm{E}} \approx 5 \Gamma_{\mathrm{O}}$. In Sec. IV, we model the interactions between nanorods as point dipoles and as finite-size cylinders using this value. In Secs. IV B and IV C, where the length of the nanorod is important, the resonance frequency of each nanorod is assumed to depend on the length of the rod as shown in Fig. 12.

\section{APPENDIX B: ASYMMETRIC COUPLING OF COLLECTIVE MODES}

In Sec. IV C, we showed how linearly-polarized light could drive a toroidal dipole response in a metamolecule whose constituent rods vary in resonance frequency. We found an optimal variation in the limit that the resonance frequency shifts were much larger than the rod linewidths, and interactions between individual rods can be neglected. In this Appendix, we show how this scheme also works in the presence of interactions. Describing the evolution of the system in terms of collective eigenmodes of a symmetric metamolecule, we see how the introduced asymmetry couples collective metamolecule modes. We will see that a judicious combination of rod lengths can strongly couple an electric dipole mode (driven by the incident linearly-polarized light) to the toroidal dipole mode (which is invisible to the incident field without rod asymmetries).

Formally, the evolution of the resonator excitations can be expressed in terms of the driven, system of equations Eq. (7). When all rods are of equal length $H_{j}=H_{0}$, the coupling matrix $\mathcal{C}=\mathcal{C}_{\text {sym }}$, and we denote the driving of the resonators as $\mathbf{F}_{\text {sym }}$, with elements $F_{\text {sym }, j}$ given in Eq. (18). As discussed in Sec. IV A, this symmetric metamolecule has eigenmodes of oscillation (labelled by index $n$ ) corresponding to eigenvectors $\mathbf{v}_{n}$ of $\mathcal{C}_{\text {sym }}$ and eigenvalues $\xi_{n}=-i \delta_{n}-\gamma_{n} / 2$, where $\delta_{n}$ is the shift of the collective mode resonance frequency from the reference $\Omega_{0}$, and $\gamma_{n}$ is the collective decay rate, Eq. (16). Generally, any metamolecule excitation $b$ can be expressed as

$$
\mathbf{b}=\sum_{n} c_{n}(t) \mathbf{v}_{n}=\mathcal{S} \mathbf{c},
$$

where $\mathcal{S}$ is a matrix whose $n$th column is the eigenvector $\mathbf{v}_{n}$ of $\mathcal{C}_{\text {sym }}$, and $\mathbf{c} \equiv\left(c_{1}, \ldots, c_{n}\right)^{T}$. The electric dipole $\mathbf{v}_{\mathrm{E} 1}$ and toroidal dipole $\mathbf{v}_{\mathrm{t}}$ excitations are eigenmodes of the symmetric metamolecule, where $\mathbf{v}_{\mathrm{E} 1}$ is directly driven by the incident field. When the two layers of rods are separated by much less than a wavelength, the incident field drives only one of the two modes where the adjacent dipoles oscillate symmetrically, leaving all of the other modes, including the toroidal dipole unexcited.

Here we generalize the treatment of Sec. IV B, which dealt with the limit of noninteracting rods, to show how introducing an asymmetry into the rod lengths can lead to the excitation of a toroidal dipole. Perturbing the lengths of each rod $j$ by $\delta H_{j}$ during the fabrication, alters the coupling matrix $\mathcal{C}$. As discussed in Sec. IV B, the primary consequence of altering the rod lengths is that each rod has its resonance frequency shifted by $\delta \omega_{j}$ proportional to $\delta H_{j}$, as indicated in Fig. 12. Additionally, changing rod lengths impacts the interactions between metamolecules. We denote the deviation of the coupling matrix from that for the symmetric system as

$$
\mathcal{A} \equiv \mathcal{C}-\mathcal{C}_{\text {sym }}
$$

From Eq. (7), the amplitude of each amplitude $c_{n}$ in the expansion of Eq. (B1) obeys

$$
\dot{\mathbf{c}}=\left[\Lambda+\mathcal{S}^{-1} \mathcal{A S}\right] \mathbf{c}+\mathbf{f}+\delta \mathbf{f},
$$

where $\mathbf{f} \equiv \mathcal{S}^{-1} \mathbf{F}_{\text {sym }}$ is the vector of driving amplitudes for each individual mode, and $\Lambda$ is the diagonal matrix of eigenvectors of $\mathcal{C}_{\text {sym }}$. Changing the rod lengths also alters the driving by $\delta \mathbf{f} \equiv \mathcal{S}^{-1} \delta \mathbf{F}$, where $\delta F_{j}=\delta F_{j} \cos \theta_{j}$ is the change in driving amplitude experienced by $\operatorname{rod} j$, and $\delta F_{j}$ is the change in driving amplitude a rod would experience if it were oriented along the incident field polarization, as discussed in Sec. IV B. Essentially, altering the lengths of the rods induces coupling between the eigenmodes via the asymmetry matrix $\mathcal{S}^{-1} \mathcal{A S}$. At the same time, while a linearly-polarized incident field only drives electric dipole modes in the symmetric metamolecule, a nonzero $\delta \mathbf{f}$ introduced by the asymmetry permits other modes to be driven directly by the incident field.

As in Sec. IV B, our goal in altering the lengths is to find a perturbation that permits the excitation of the toroidal dipole mode, while reducing the contribution of other collective modes. In particular, consider the steady-state excitation induced by a field resonant on the toroidal dipole of the symmetric system

$$
\mathbf{c}=-\left[\Lambda+i \delta_{\mathrm{t}}+\mathcal{S}^{-1} \mathcal{A S}\right]^{-1}(\mathbf{f}+\delta \mathbf{f}),
$$

where the subscript " $t$ " refers to the toroidal dipole mode. Since altering the lengths of the rods induces coupling between the modes, one can produce a toroidal dipole excitation by introducing a coupling between the toroidal dipole mode and other modes in the metamolecule, in particular the electric dipole mode. In general, one would obtain the optimal excitation of the toroidal dipole by optimizing the rod length perturbations $\delta H_{j}$. The general optimization procedure would account for changes in interactions between resonators produced by the asymmetry as well as the interactions that are present in the symmetric metamolecule.

Here, we illustrate how rod lengths would be chosen when the only effect of $\delta H_{j}$ is to produce changes in resonance frequencies $\delta \omega_{j}=\chi \delta H_{j}$ and individual rod decay rates $\delta \Gamma_{j}=\nu \delta H_{j}$ in proportion to $\delta H_{j}$ for some constants $\chi$ and $v$. In this case, the matrix $\mathcal{A}=\operatorname{diag}\left(-\delta \omega_{1}-\delta \Gamma_{1} / 2, \ldots,-i \delta \omega_{N}-\delta \Gamma_{N} / 2\right)$. We will also assume the separation between rod layers is much less than a wavelength so that each layer experiences an identical driving. In these limits, we ask the question: what conditions would have to be satisfied to have the steady-state response of the metamolecule to be purely in the toroidal dipole mode? From there, we deduce a combination of $\delta H_{j}$, that could yield a toroidal dipole response. Consider an excitation of the form $\mathbf{b}=c_{0} \mathbf{v}_{\mathrm{t}}$ at time $t=t_{0}$, entirely in the toroidal dipole mode. Then, from Eqs. (7), (18), and (19), we have, for each resonator $j$,

$$
\begin{aligned}
\left.\frac{d}{d t} b_{j}\right|_{t=t_{0}}= & -c_{0} \frac{\gamma_{\mathrm{t}}}{2} \mathbf{v}_{\mathrm{t}}(j)-i c_{0}(\chi-i \nu) \delta H_{j} \mathbf{v}_{\mathrm{t}}(j) \\
& +\left(F_{0}+\delta F_{j}\right) \cos \theta_{j} .
\end{aligned}
$$


From Eq. (B4), we see that if $\gamma_{\mathrm{t}}$ is negligible, i.e., $\gamma_{\mathrm{t}} \ll \delta \omega_{j}$, $\Gamma$, the toroidal excitation is in the steady state when for each nanorod $j, \delta H_{j}$ solves

$$
i c_{0}(\chi-i \nu) \delta H_{j} \mathbf{v}_{\mathrm{t}}(j)=\left(F_{0}+\delta F_{j}\right) \cos \theta_{j} .
$$

As in Sec. IVB, where we considered noninteracting metamolecules, to lowest order in $\delta H_{j}$, the asymmetry in rod lengths needed to generate a toroidal dipole is given by Eq. (22).

Thus, when the collective decay rate $\gamma_{\mathrm{t}} \ll \delta \omega_{j}$, and we have neglected how the asymmetry of rod lengths alters interactions between the rods, the asymmetry of Eq. (22) would yield a toroidal dipole amplitude

$$
c_{0}=-i \sqrt{N} \frac{F_{0}}{(\chi-i v) \delta H_{0}} .
$$

This is remarkably similar in form to the toroidal dipole amplitude one would obtain if one neglected all interactions between nanorods as done in Sec. IV B. In accounting for interactions, we no longer need to assume that $\delta \omega_{j} \gg \Gamma$. One does, however, need to drive the metamolecule with a field resonant on the toroidal dipole mode of the symmetric metamolecule, rather than resonant with a single nanorod.
[1] Ya. B. Zel'dovich, Zh. Eksp. Teor. Fiz. 33, 1531 (1957) [Sov. Phys. JETP 6, 1184 (1958)].

[2] E. E. Radescu and G. Vaman, Phys. Rev. E 65, 046609 (2002).

[3] W. C. Haxton, Science 275, 1753 (1997).

[4] V. V. Flambaum and D. W. Murray, Phys. Rev. C 56, 1641 (1997).

[5] V. F. Dmitriev and I. B. Khriplovich, Phys. Rep. 391, 243 (2004).

[6] V. V. Flambaum and I. B. Khriplovich, Zh. Eksp. Teor. Fiz. 79, 1656 (1980) [Sov. Phys. JETP 52, 835 (1980)].

[7] H. Schmid, Ferroelectrics 252, 41 (2001).

[8] C. Ederer and N. A. Spaldin, Phys. Rev. B 76, 214404 (2007).

[9] A. Politano and G. Chiarello, Front. Mater. 1, 9 (2014).

[10] S. Hayami, H. Kusunose, and Y. Motome, Phys. Rev. B 90, 024432 (2014).

[11] V. Dubovik and V. Tugushev, Phys. Rep. 187, 145 (1990).

[12] V. M. Dubovnik and A. A. Cheskov, Fiz. Elem. Chast. At. Yadra 5, 791 (1974) [Sov. J. Part. Nucl. Phys. 5, 318 (1974)].

[13] E. E. Radescu and D. H. Vlad, Phys. Rev. E 57, 6030 (1998).

[14] J. D. Jackson, Classical Electrodynamics (Wiley, New York, 1999).

[15] Morse and Feshbach, Methods of Theoretical Physics (McGraw-Hill, New York, 1953).

[16] L. Landau and E. Lifshitz, The Classical Theory of Fields, (Pergamon, Amsterdam, 1975).

[17] A. Gongora and E. Ley-Koo, Rev. Mex. Fis. E 52(2), 188 (2006).

[18] V. A. Fedotov, A. V. Rogacheva, V. Savinov, D. P. Tsai, and N. I. Zheludev, Sci. Rep. 3, 2967 (2013).

[19] Y. Fan, Z. Wei, H. Li, H. Chen, and C. M. Soukoulis, Phys. Rev. B 87, 115417 (2013).

[20] Q. W. Ye, L. Y. Guo, M. H. Li, Y. Liu, B. X. Xiao, and H. L. Yang, Phys. Scr. 88, 055002 (2013).

[21] T. Kaelberer, V. A. Fedotov, N. Papasimakis, D. P. Tsai, and N. I. Zheludev, Science 330, 1510 (2010).

[22] Z.-G. Dong, J. Zhu, J. Rho, J.-Q. Li, C. Lu, X. Yin, and X. Zhang, Appl. Phys. Lett. 101, 144105 (2012).

[23] Y.-W. Huang, W. T. Chen, P. C. Wu, V. Fedotov, V. Savinov, Y. Z. Ho, Y.-F. Chau, N. I. Zheludev, and D. P. Tsai, Opt. Express 20, 1760 (2012).

[24] Y.-W. Huang, W. T. Chen, P. C. Wu, V. A. Fedotov, N. I. Zheludev, and D. P. Tsai, Sci. Rep. 3, 1237 (2013).

[25] V. Savinov, V. A. Fedotov, and N. I. Zheludev, Phys. Rev. B 89, 205112 (2014).

[26] Z.-G. Dong, J. Zhu, X. Yin, J. Li, C. Lu, and X. Zhang, Phys. Rev. B 87, 245429 (2013).
[27] Z.-G. Dong, P. Ni, J. Zhu, X. Yin, and X. Zhang, Opt. Express 20, 13065 (2012).

[28] H. Wang, E. Yan, E. Borguet, and K. Eisenthal, Chem. Phys. Lett. 259, 15 (1996).

[29] B. K. Canfield, S. Kujala, H. Husu, M. Kauranen, B. Bai, J. Laukkanen, M. Kuittinen, Y. Svirko, and J. Turunen, J. Nonlinear Opt. Phys. Mater. 16, 317 (2007).

[30] S. A. Maier and H. A. Atwater, J. Appl. Phys. 98, 011101 (2005).

[31] S. D. Jenkins and J. Ruostekoski, Phys. Rev. B 86, 085116 (2012).

[32] S. D. Jenkins and J. Ruostekoski, New J. Phys. 14, 103003 (2012).

[33] S. D. Jenkins and J. Ruostekoski, Phys. Rev. Lett. 111, 147401 (2013).

[34] G. Adamo, J. Y. Ou, J. K. So, S. D. Jenkins, F. De Angelis, K. F. MacDonald, E. Di Fabrizio, J. Ruostekoski, and N. I. Zheludev, Phys. Rev. Lett. 109, 217401 (2012).

[35] H. Kuwata, H. Tamaru, K. Esumi, and K. Miyano, Appl. Phys. Lett. 83, 4625 (2003).

[36] B. Schrader, Infrared and Raman Spectroscopy: Methods and Applications (Wiley-VCH Verlag GmbH, Weinheim, Germany, 2007).

[37] Note that the special case $n=4$ has the symmetry group $D_{4 h}$.

[38] S. D. Jenkins and J. Ruostekoski, Phys. Rev. B 86, 205128 (2012).

[39] B. Luk'yanchuk, N. I. Zheludev, S. A. Maier, N. J. Halas, P. Nordlander, H. Giessen, and C. T. Chong, Nat. Mater. 9, 707 (2010).

[40] M. Fleischhauer, A. Imamoglu, and J. P. Marangos, Rev. Mod. Phys. 77, 633 (2005).

[41] M. Hentschel, D. Dregely, R. Vogelgesang, H. Giessen, and N. Liu, ACS Nano 5, 2042 (2011).

[42] C. Bohren and D. Huffman, Absorption and Scattering of Light by Small Particles (Wiley-VCH Verlag GmbH \& Co. KGaA, Weinheim, Germany, 2004).

[43] V. I. Emelyanov, Laser Phys. 8, 937 (1998).

[44] V. I. Emelyanov, E. M. Zemskov, and V. N. Seminogov, Phys. Chem. Mech, Surfaces 3(2), 381 (1985).

[45] P. B. Johnson and R. W. Christy, Phys. Rev. B 6, 4370 (1972).

[46] E. J. Zeman and G. C. Schatz, J. Phys. Chem. 91, 634 (1987).

[47] N. Grady, N. Halas, and P. Nordlander, Chem. Phys. Lett. 399, 167 (2004). 